\title{
Case Report: Lead Poisoning Can Be Misdiagnosed as Guillain-barre Syndrome: A Case Report
}

\author{
Abbas Aghabiklooei ${ }^{1}$, Maryam Ameri $^{\mathbf{1}^{*}} \mathrm{Q}$ \\ 1. Firoozgar Hospital, Iran University of Medical Sciences, Tehran, Iran.
}

\begin{tabular}{|c|c|}
\hline $\begin{array}{l}\text { Use vourd device toscsan } \\
\text { and read the artice online }\end{array}$ & Chtation: Aghabiklooei A, Ameri M. Lead Poisoning Can Be Misdiagnosed as Guillain-barre Syndrome: A Case Report \\
\hline 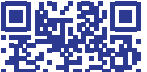 & $\begin{array}{l}\text { International Journal of Medical Toxicology and Forensic Medicine. 2019; 9(3):171-174. https://doi.org/10.32598/ijmtfm. } \\
\text { v9i3.22916 }\end{array}$ \\
\hline latizis & https://doi.org/10.32598/ijmtfm.v9i3.22916 \\
\hline
\end{tabular}

\section{(A) 0}

Article info:

Received: 17 Jan 2019

First Revision: 11 Feb 2019

Accepted: 13 May 2019

Published: 01 Jul 2019

\section{Keywords:}

Guillain-Barre syndrome, Lead Poisoning, Toxic neuropathy

\begin{abstract}
A B S T R ACT
Background: Toxic neuropathy is a global health problem affecting many people over the world, annually. Lead Poisoning (LP) represents neurological complaints and neurobehavioral disorders. Therefore, its significances, especially its neurological consequences, can be misdiagnosed as other neuropathies like syndromes.

Case Presentation: In this case report, we aim at describing a 45-year-old woman with Guillain-Barre-like Syndrome (GBS), who was admitted to the hospital with peripheral neuropathy and complaints of paresthesia in the lower and upper limbs. Suffering from GBSlike syndrome, she incidentally showed opium consumption during the hospitalization and taking her history. Serum lead levels were precisely elevated $(88.6 \mu \mathrm{g} / \mathrm{dL})$. To assess the neurologic effects, the brain CT, MRI, EMG, and NCV were performed, indicating severe sensory-motor demyelinating polyradiculopathy. The patient was admitted to the hospital again after 2 years with severe radicular pain in the lower and upper limbs and with positive myoclonus and tremor. While treated with plasma exchange and pregabalin at the first stage of admission, signs were normal and again recurred after 2 years, suggesting the reversibility of the histological findings and misdiagnosis. At the second stage, Intravenous Immunoglobulin (IVIG) was prescribed. The patient recovered and was discharged with chelation therapy of CaNa2EDTA for LP. The most frequent neurological complication induced by LP is severe sensory-motor demyelinating polyradiculopathy and axonal polyneuropathy. But, the clinical examination and the electrophysiological findings may also suggest a GBS-like syndrome.
\end{abstract}

Conclusion: Any discrepancies in this regard should be reconsidered to confirm LP diagnosis.

\section{Introduction}

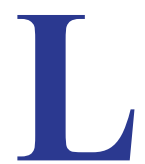

ead Poisoning (LP) is manifested with a variety of symptoms depending on the lead exposure duration $[1,2]$. Toxic neuropathies are often misdiagnosed as other disorders because of the lack of available specific or biological diagnostic tools [3]. The previously published study had presented a case of LP in a 5-year-old girl with the signs of anemia, encephalopathy, and peripheral neuropathy mimicking GBS [2]. Another study had reported a subacute arsenical neuropathy leading to GBS-like and respiratory failure [4]. GBS-like syndrome and encephalopathy have also

* Corresponding Author:

Maryam Ameri, PhD.

Address: Firoozgar Hospital, Iran University of Medical Sciences, Tehran, Iran.

Tel: +98 (912) 7193692

E-mail: maryam.ameri12@yahoo.com 
been observed in a 50-year-old man after acute arsenical poisoning [5]. GBS is a complicated degenerative neurological disorder with muscle weakness associated with peripheral neuropathy as a commonly encountered condition in clinical practice. Therefore, the differential diagnosis of GBS from other toxic neuropathies is mostly important. Here, we report a 45-year-old woman with GBS-like syndrome, who was diagnosed with LP. The present case study highlights the difficulty in diagnosing heavy metal intoxication in patients presenting with peripheral neuropathy.

\section{Case Report}

A 45-year-old woman was admitted to the hospital with the complaints of paresthesia in the lower and upper limbs. Two weeks before admission, she had limb weakness and could not walk well.

The clinical and neurological examination were performed at the time of admission with the following pathological symptoms: normal sensory, lower limb atrophy, DTR 1+, plantar reflex, down-word with steppage gait, and anemia with an unknown cause.

The patient was admitted to the hospital with GBS diagnosis on September 13, 2014. Then, the following evaluations were performed: 1. CXR and Brain CT: Normal; 2. Brain MRI: Decreased CAMP amplitudes in all examined motor nerves. SNAP in all examined sensory nerves. Prolonged motor DL in all examined nerves. Absent bilateral common peroneal CAMPs; 3. EMG and $\mathrm{NCV}$ : severe sensory-motor demyelinating polyradiculopathy; 4. EEG and lumbar puncture: Normal; 5. Abdominal and pelvic ultrasound: Normal

The treatment began with several sessions of plasma exchange after the admission. The patient recovered and was discharged with a dose of 4 pregabalin prescription (150 mg daily).

Two years after discharge, the patient was admitted to the hospital on April 3, 2016, with severe radicular pain in the lower limb. Then, weakness in the proximal side of the lower limb was manifested. A week later, pain in the upper limb and in 2 sides of wrist drop appeared. Other complaints manifested in the patient were positive myoclonus and tremor.

The patient was suspected of Guillen-Barre syndrome and was admitted. A proven effective treatment for GBS (class 1 evidence), IVIG, was then prescribed. During the hospitalization and taking the patient's history, we found that the patient has orally consumed opium. The lead blood level was requested and we found a high level of lead $(88.6 \mu \mathrm{g} / \mathrm{dL})$ in the patient serum sample.

In the second stage, we found that her problem was LP. The patient was treated with chelation therapy of CaNa2EDTA and a significant improvement was observed in the patient's complaints such as paresthesia, weakness, and wrist drop.

In the second stage, the following evaluations were performed: 1. MRI and Brain CT: Normal; 2. EMG and NCV: Subacute axonal sensory-motor polyneuropathy. The patient recovered and was discharged with pregabalin and Deakin prescription.

\section{Discussion}

The most common sign of LP in adults is peripheral neuropathy [2]. It has been shown that acute neuropathy is initially misdiagnosed as GBS [6]. To identify the potential sources of lead exposure, taking a medical and environmental history, looking for signs on physical examination, and confirming excessive lead exposure or organ system damage looking for laboratory evidence are required [7]. The first main step in eliminating the signs of the disease is the reduction and removal of lead exposure [7]. Here, we report a 45-year-old woman, who was diagnosed with GBS-like neuropathy associated with lead exposure and poisoning. Fatemi et al. reported acute LP in an opium user as a much uncommon source of exposure through the inhalation and oral addiction [7]

In their report, abdominal pain, nausea and vomiting, severe weight loss, generalized bone pain, and jaundice were observed as common signs of the patient. In the current study, we have reported a case, who was referred to hospital and treated with GBS symptoms; but in the second referral, we found that her problem was LP of oral opium consumption. LP among Iranian opium users was reported in less than a decade ago $[8,9,10]$. In a review performed on the Iranian cases of LP from opium abuse in 2009, the most common symptoms and signs of cases were abdominal pain, nausea, and abdominal tenderness, icter, and neurologic problem, respectively. In all cases, anemia and elevated liver enzymes (ALT and AST) were the common features in laboratory tests [11]

The users of opium products may be at the risk of heavy metal toxicity, especially lead; therefore, the diagnosis of opium user should be mandatory [1]. On the other hand, the differential diagnosis of LP, which can imitate the symptoms of GBS because of peripheral neu- 
ropathy, is highly recommended in the clinics. In our study, the patient was discharged and treated without any complaints after the second time of admission, and we recommended no further exposure to lead products. In several studies in Iran, anemia had been reported as the main clinical manifestation among the opium users.

It is stated that most of the opium in the black market of Iran is contaminated with lead; we request drug users to stop opium immediately in order to initiate their treatment in the clinics [12]. In our study, we found that the patient had orally consumed opium during the hospitalization and taking her history. Multiple sclerosis-like symptom is another feature of lead exposure mimicking LP with the elevation of serum lead and zinc protoporphyrin levels 17 in opium users.

\section{Conclusion}

Several characteristics of LP are considered a differential diagnosis of LP from other toxic neuropathies. One of the major problems regarding the LP is a delay in its recognition for long periods of time because of a low index of suspicion. Therefore, it is essential to consider the differential diagnosis of any unexplained GBS-like symptoms, especially in opium addicts. The evaluation of the lead concentration in a serum sample of opium addicts with GBS-like symptoms is highly recommended.

\section{Ethical Considerations}

\section{Compliance with ethical guidelines}

Demographic information taken from the patient's case is considered confidential.

\section{Funding}

This research did not receive any specific grant from funding agencies in the public, commercial, or not-forprofit sectors.

\section{Author's contributions}

All authors contributed in preparing this article.

\section{Conflict of interest}

The authors declared no conflict of interest with respect to this manuscript.

\section{References}

[1] Mărginean CO, Meliț LE, Moldovan H, Lupu VV, Mărginean MO. Lead poisoning in a 16-year-old girl: A case report and a review of the literature (CARE compliant). Medicine. 2016 95(38):e4916. [DOI:10.1097/MD.0000000000004916] [PMID] [PMCID]

[2] Toto M, De Giacomo A, Petruzzelli MG, Dicuonzo F Santoro N, Del Vecchio GC, et al. Guillain-Barré-Like syndrome in a child with lead poisoning. Neuropediatrics. 2012; 43(04):217-20. [DOI:10.1055/s-0032-1321981] [PMID]

[3] Misra UK, Kalita J. Toxic neuropathies. Neurology India. 2009; 57(6):697-705. [DOI:10.4103/0028-3886.59463] [PMID]

[4] Singh S, Reddy S, Sud A, Wanchu A, Bambery P, Gill K. Subacute arsenical neuropathy leading to Gullian-Barre-like syndrome and respiratory failure. Journal of the Association of Physicians of India. 2002; 50:853-4. [PMID]

[5] Perriol M, Devos D, Hurtevent J, Tiffreau V, Saulnier F, Destee A, et al. A case of neuropathy mimicking Guillain-Barre syndrome after arsenic intoxication. Revue Neurologique. 2006; 162(3):374-7. [DOI:10.1016/S0035-3787(06)75025-1]

[6] Kim S, Takeuchi A, Kawasumi Y, Endo Y, Lee H, Kim Y A Guillain-Barré syndrome-like neuropathy associated with arsenic exposure. Journal of Occupation Health. 2012; 54(4):344-7. [DOI:10.1539/joh.12-0023-CS] [PMID]

[7] Fatemi R, Jafarzadeh F, Moosavi S, Amin FA. Acute lead poisoning in an opium user: A case report. Gastroenterology and Hepatology from bed to bench. 2008; 1(3):139-42. [DOI:10.22037/ghfbb.v1i3.36]

[8] Masoodi M, Zali MR, Ehsani-Ardakani MJ, Mohammad-Alizadeh AH, Aiassofi K, Aghazadeh R, et al. Abdominal pain due to lead-contaminated opium: A new source of inorganic lead poisoning in Iran. Archives of Iranian Medicine. 2006; 9(1):72-5. [PMID]

[9] Aghaee-Afshar M, Khazaeli P, Behnam B, Rezazadehkermani M, Ashraf-Ganjooei N. Presence of lead in opium. Archives of Iranian Medicine. 2008; 11(5):553-4. [PMID]

[10] Jalili M, Azizkhani R. Lead toxicity resulting from chronic ingestion of opium. The Western Journal of Emergency Medicine; 2009; 10(4):244-6. [PMID]

[11] Karimi G, Moshiri M, Etemad L. Review of cases of lead poisoning from opium abuse in Iran. Pharmacologyonline. 2009; 3:897-905.

[12] Radfar SR, Nematollahi P, Farhoudian A, Noroozi A Lead Poisoning among Opium Users in Iran, a Possible New Emerging Epidemic in the Region. Iran Journal of Public Health. 2017; 46(8):1152-3. [PMID] 
This Page Intentionally Left Blank 